\title{
Orthogonal Synthesis of Highly Porous Zr-MOFs Assembled from Simple Building Blocks for Oxygen Storage
}

\author{
Jiafei Lyu, ${ }^{\dagger, £, \S ~ X u a n ~ Z h a n g, § ~ Z h i j i e ~ C h e n, § ~ R y t h e r ~ A n d e r s o n, " X i n g j i e ~ W a n g, § ~ M e g a n ~ C . ~ W a s s o n, § ~ P e n g ~}$ \\ Bai, ${ }^{\dagger, \ddagger}$ Xianghai Guo, ${ }^{\dagger} \neq$ Timur Islamoglu, ${ }^{\S}$ Diego A. Gómez-Gualdrón," and Omar K. Farha*,,\# \\ †Department of Pharmaceutical Engineering, School of Chemical Engineering and Technology, Tianjin University, \\ Tianjin 300350, China \\ ¥Key Laboratory of Systems Bioengineering, Ministry of Education, Tianjin University, Tianjin 300350, China \\ §Department of Chemistry and International Institute of Nanotechnology, Northwestern University, 2145 Sheridan \\ Road, Evanston, Illinois 60208, United States \\ "Department of Chemical and Biological Engineering, Colorado School of Mines, Golden Colorado 80401, United States \\ \#Department of Chemical and Biological Engineering, Northwestern University, 2145 Sheridan Road, Evanston, \\ Illinois 60208, United States \\ Supporting Information Placeholder
}

\begin{abstract}
The last decade has witnessed significant advances in the scale-up synthesis of metal-organic frameworks (MOFs) using commercially available and affordable organic linkers. However, the synthesis of MOFs using elongated and/or multitopic linkers to access MOFs with large pore volume and/or various topologies can often be challenging due to multi-step organic syntheses involved for linker preparation. In this report, a orthogonal MOF synthesis strategy is developed by utilizing the coordination and covalent bonds formation in one-pot where monoacid-based ligands reacted to form ditopic ligands which then assembled into a 3-D MOF with $\mathrm{Zr}_{6}$ clusters. Chemical stability of the resulting materials was significantly enhanced through converting the imine bond into robust linkage via cycloaddition with phenylacetylene. Oxygen storage capacities of the MOFs were measured, and enhanced volumetric $\mathrm{O}_{2}$ uptake was observed for the stabilized MOF, NU-401-Q.
\end{abstract}

KEYWORDS: Zr-MOFs, one-pot syntheses, Imine bonds, stabilization, $\mathrm{O}_{2}$ uptake

\section{Introduction}

Metal-organic frameworks (MOFs) ${ }^{1-4}$ are a class of crystalline porous materials, constructed from metal ions or clusters and organic ligands, with reported record-high internal surface areas. 5,6 They are highly attractive due to their remarkable performance in various applications including, adsorption/separation, ${ }^{7-15}$ catalysis, ${ }^{16-18}$ drug delivery ${ }^{19,20}$ and sensors. ${ }^{21,22}$. With the metal nodes as the joints, the organic linkers serve as the spacer which plays a crucial role in dictating the topology and properties of the resulting MOFs. This versatile structural tunability has inspired extensive research to develop MOFs with different chemical and physical properties by simply varying the organic linkers or the strategic implemenation of reticular chemistry, ${ }^{23}, 24$ post-synthetic modification ${ }^{25}, 26$ and ligand exchange/insertion. ${ }^{27}$

The development of multitopic organic ligands with various functionalities and geometries is essential for the discovery of MOFs with novel properties and topologies. ${ }^{28}$ For those sophisticated ligands with intriguing structures and functionalities, the syntheses typically involve multi-step organic reactions, which could be laborious and costly due to the increasing demand of time and reagents. As a result, cost and time efficient syntheses of linkers are envisioned to better faciliate the scale-up syntheses of MOFs constructed by intricate organic linkers.

Pioneering research has been implemented toward the simplification of MOF preparation procedures where a traditional de novo synthesis is challenging. For example, post-synthetic metathesis and cyclization reactions have been successfully utilized to generate fused aromatic systems in MOFs. ${ }^{29}, 30$ Additionally, a combination of in situ linker syntheses and solvothermal MOF syntheses was adopted for one-pot MOF assembly. 31,32 Yaghi and coworks reported a 2-D titanium-organic framework with imine ligands in situ condensated. ${ }^{33}$ Such strategies have demonstrated cyclisation, ${ }^{34}$ dehydrogenative or oxidative coupling, ${ }^{35}, 36$ hydroxylation, ${ }^{35}, \quad 37$ hydrolysis, ${ }^{38-40}$ cleavage of the $S-S$ bonds, ${ }^{41,} 42$ imine condensation, ${ }^{33,43}$ cycloaddition of organic nitriles ${ }^{44,}$ 45 and $\mathrm{C}-\mathrm{C}$ heterocoupling; in the latter case amorphous hybrid porous materials were produced. ${ }^{46}$ More recently, diazo coupling, imidizatio and condensation of boronic acids ${ }^{47}$ were also reported to be candidates for one pot strategy during the preparation of this manuscript. Although the synthesis procedures were simplified to a one-pot strategy where both organic ligand syntheses and 
MOF assembly occurs spontenously, the introduction of additional reagents for desired ligand formation complicated the synthesis which could ultimately compromise the crystallinity and porosity of resulting networks.

Zirconium based-MOFs (Zr-MOFs), constructed from $\mathrm{Zr}_{6}$ clusters $\left(\mathrm{Zr}_{6}\left(\mu_{3}-\mathrm{O}\right)_{4}\left(\mu_{3}-\mathrm{OH}\right)_{4}\right)$ and multitopic organic linkers, have been extensively investigated due to their exceptional stability, topological diversity and ease of preparation. ${ }^{23}$ While there are many efforts for aqueous solution based preparation of ZrMOFs, their economically viable preparation would be greatly benefited from a one-pot synthesis strategy. To this end, it was hypothesized that Schiff base imine $(\mathrm{C}=\mathrm{N})$ based linkers, in which aldehyde and amine based building blocks could condense under solvothermal conditions, would be advantegous since their condensation product can form under similar conditions as MOF syntheses require.

In this report, with dynamic covalent imine bonds and coordination bonds between $\mathrm{Zr}_{6}$ clusters and carboxylates, two highly porous Zr-MOFs with fcu topology were synthesized via one-pot reactions by the in situ assembly of imine-based ligands. Furthermore, the intrinsically reversible imine bonds in the resulting network were successfully transformed into stable quinoline structures by the Aza-Diels-Alder cycloaddition reaction, which dramatically enhanced the hydrolytic stability over the parent structure. Motivated by the high porosity of the resulting MOFs, $\mathrm{O}_{2}$ storage capacity of the materials reported in this study was also examined.

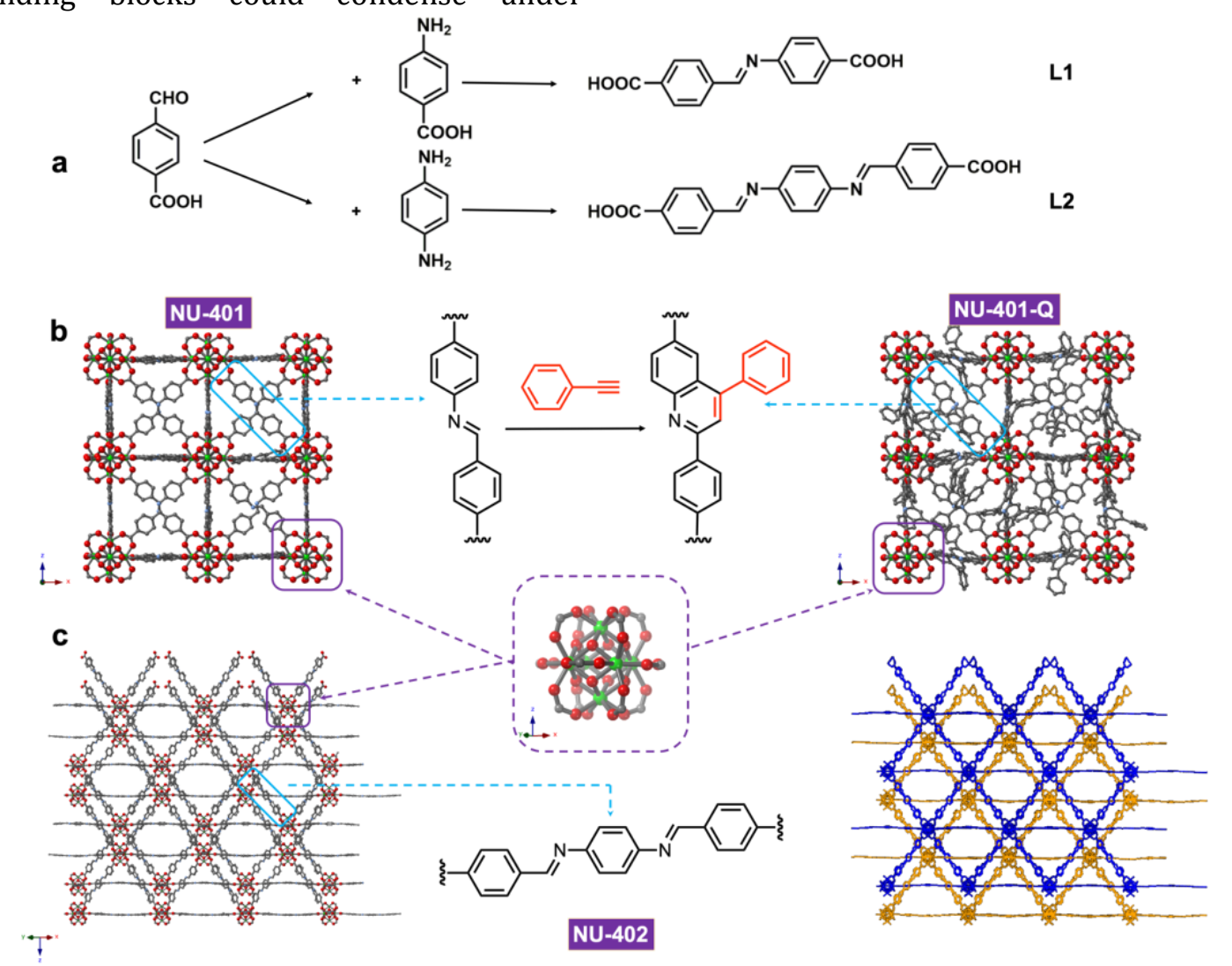

Figure 1. Schematic representation of (a) the formation of imine bonds with aldehyde and amine groups, (b) NU-401 (left), NU401-Q (right) and ligand stabilization reaction (middle), (c) catenated $\mathbf{N U}-\mathbf{4 0 2}$ and $\mathrm{Zr}_{6}$ cluster (middle). Lime green $=\mathrm{Zr}$, Blue $=$ $\mathrm{N}$, Gray $=\mathrm{C}$, Red $=0$.

\section{Results and Discussion}

By manipulating the ratios of starting precursors and reaction conditions in one-pot syntheses from simple monocarboxylates with amino- and aldehyde groups, two fcu-net MOFs were synthesized with high crystallinity, remarkable porosity and extended apertures (Figure 1). NU-401 with $\mathbf{L 1}$ as the organic linker was crystallized from one-pot solvothermal reaction, where the inexpensive and readily available 4-aminobenzoic acid and 4-formylbenzoic acid were employed as the building blocks for an in situ organic ligand assembly. Moreover, the monocarboxylate moities served as modulators for the coordination reactions between the dicarboxylate linkers and $\mathrm{Zr}_{6}$ clusters. The crystallinity of NU-401 was observed from the experimental powder X-ray diffraction (PXRD) pattern (Figure 2, up), through which the crystal structure of NU-401 was constructed using the ToBaCCo software for computational MOF synthesis according to topological blueprints by GómezGualdrón and coworkers. ${ }^{48,} 49$ The structure output from ToBaCCo was geometrically optimized according 
to the UFF50 using the Forcite Module of Materials Studio $^{51}$. The structure of NU-401 was further confirmed via Pawley refinement of the experimental PXRD data, and the refinement results $\left(R_{w}=6.2 \%\right.$, Table S1 and Figure S4) matched well with the optimized unit cell parameters. Uniform octahedral particles $(\sim 1 \mu \mathrm{m}$, Figure S1) were observed by Scanning Electron Microscopy (SEM). The internal porosity was characterized by nitrogen adsorption/desorption measurements under $77 \mathrm{~K}$ where type I isotherms were observed (Figure 2, down). With the Brunauer-Emmett-Teller (BET) method, high porosity was calculated for NU-401 with apparent surface area and pore volume of $3310 \mathrm{~m}^{2} / \mathrm{g}$ and $1.35 \mathrm{~cm}^{3} / \mathrm{g}$, respectively. Correspondingly, a micropore of $1.4 \mathrm{~nm}$ was observed in the DFTcalculated pore size distribution (Figure S2), which was consistent with the size of the in situ assembled imine ligand (L1).
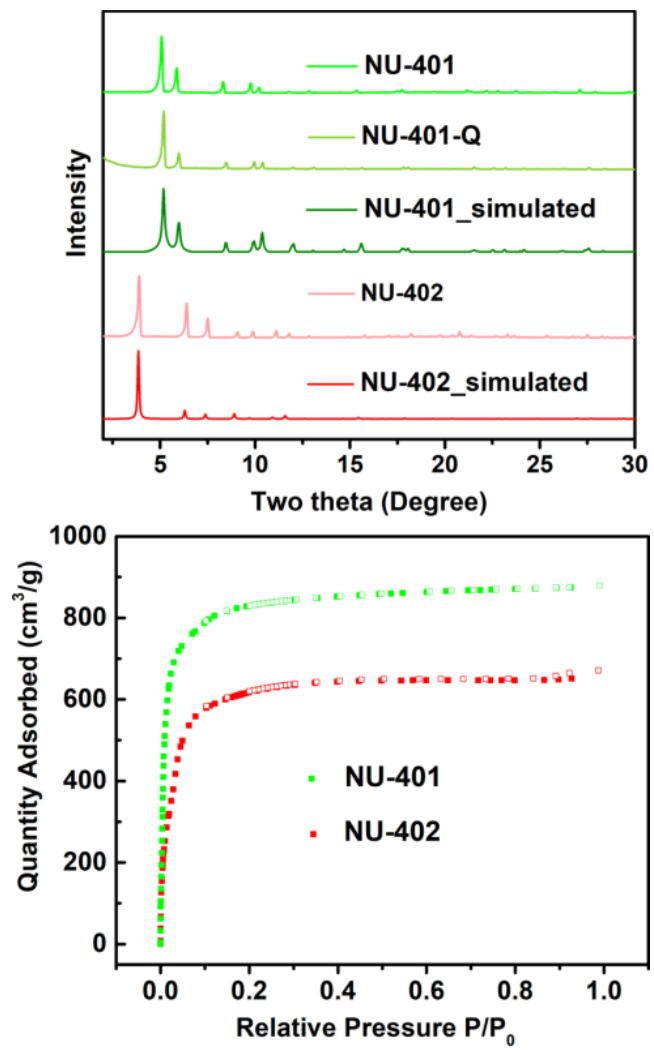

Figure 2. PXRD patterns (up) and $\mathrm{N}_{2}$ adsorption/desorption isotherms (down) of NU-401 and NU-402.

To further test our modular sythetic strategy, a diamine building block $p$-phenylenediamine, instead of 4-aminobenzoic acid, was employed together with 4-formylbenzoic acid as the assembling building blocks for an extended ditopic organic ligand. The resulting linker consisted of three phenyl rings connected by two imine bonds (L2, Figure 1) to produce the MOF NU-402. In the experimental PXRD pattern (Figure 2, up), the first peak of NU-402 appeared with a lower two theta degree than NU-401, indicating larger $\mathrm{d}$-spacing between the paralel planes due to the extended ligand. The non-interpenetrated crystal structure of NU-402 was constructed using ToBaCCo according to the fcu topology but significant inconsistency was observed between the simulated and experimental PXRD patterns. As it is known that the introduction of an extended ligand tends to increase the likelihood of structural interpenetration, ${ }^{52,53}$ an additional interpenetrated structure was created through the translation of the non-interpenetrated strucutre by half of the unit-cell in each crystallographic direction, resulting in a doubly-interpenetrated framework for NU-402 with an fcu topology. The geometry of the resulting computational model was then optimized according to the UFF using the Forcite Module of Materials Studio. The structure of NU-402 was further confirmed via Pawley refinement of the experimental PXRD data, and the refinement results $\left(R_{w}=6.7 \%\right.$, Table S1 and Figure S6) matched well with the optimized unit cell parameters. Nitrogen sorption isotherms of NU-402 showed a BET surface area of $2530 \mathrm{~m}^{2} / \mathrm{g}$ and a pore volume of $0.88 \mathrm{~cm}^{3} / \mathrm{g}$. A slightly larger micropore $(\sim 1.7 \mathrm{~nm})$ was observed by the calculation with the DFT method. Upon digestion of NU-401 and NU-402 with a diluted $\mathrm{D}_{2} \mathrm{SO}_{4} / \mathrm{d}_{6}$-DMSO solution, ${ }^{1} \mathrm{H}$ nuclear magnetic resonance (NMR) spectroscopy showed characteristic peaks for the cooresponding building blocks (Figure 3 ) due to the hydrolysis of imine bonds under acidic condition.
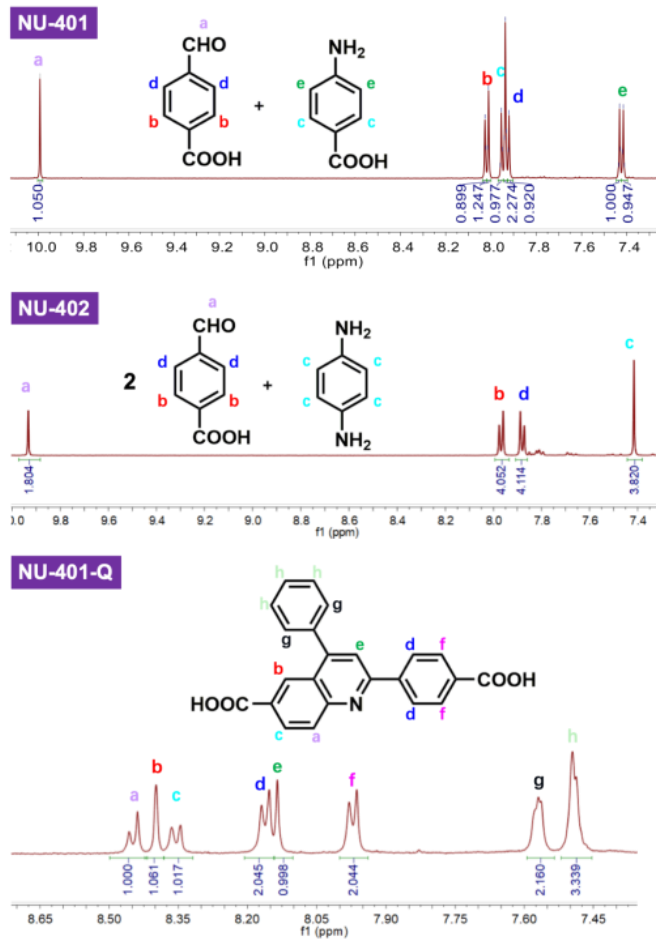

Figure 3. ${ }^{1} \mathrm{H}$ NMR spectra of NU-401 (up), NU-402 (middle), NU-401-Q (down). ( ${ }_{2} \mathrm{SO}_{4} / d_{6}-\mathrm{DMSO}, 500 \mathrm{MHz}$ ) 
Since the intrinsically reversible $\mathrm{C}=\mathrm{N}$ bond ${ }^{54}$ is subject to hydrolysis under aqueous conditions, the instability of resulting networks remains an issue. Here, postsynthetic modification (PSM) methods including reduction, ${ }^{55}$ oxidation ${ }^{56}$ and Aza-Diels-Alder addition $^{57,58}$ have been attempted to stabilize the $\mathrm{C}=\mathrm{N}$ bond, among which only the latter one with the incorporation of phenylacetylene succeeded while the MOFs degraded under the former conditions (see supporting information for details). Quinoline-linked NU-401-Q maintained its crystallinity based on the PXRD pattern after stabilization, but a one-third decrease of BET area was observed from 3310 to 2140 $\mathrm{m}^{2} / \mathrm{g}$. This was attributed to an additional phenyl group occupying more space and adding to the mass of the resulting MOF. The pore size slightly decreased from $1.4 \mathrm{~nm}$ to $1.3 \mathrm{~nm}$ due to the introduction of the bulky phenyl group. Upon digesting the NU-401-Q material, the ${ }^{1} \mathrm{H}$ NMR spectrum agreed with the corresponding structure with no signal of the original Schiff base structure, indicating complete postsynthetic functionalization (Figure 3). In addition, quinoline linkage survived from the strongly acidic digestion condition, suggesting much higher stabilily of NU-401-Q over parent NU-401. Comparison of the diffuse reflectance infrared Fourier transform spectroscopy (DRIFTS) results of NU-401 and NU401-Q revealed disappearance of a peak at $743 \mathrm{~cm}^{-1}$ representing the $\mathrm{C}-\mathrm{H}$ stretching from the $\mathrm{H}-\mathrm{C}=\mathrm{N}$ Schiff base group in the parent NU-401 instead of NU401-Q structure. Furthermore, a new set of peaks between $1600 \mathrm{~cm}^{-1}$ and $1450 \mathrm{~cm}^{-1}$ appeared which corresponded to the stretchings of the as formed aromatic quinoline core in NU-401-Q (Figure 4). As a result, the reversible $\mathrm{C}=\mathrm{N}$ bond was fixed with the introduction of phenylacetylene.

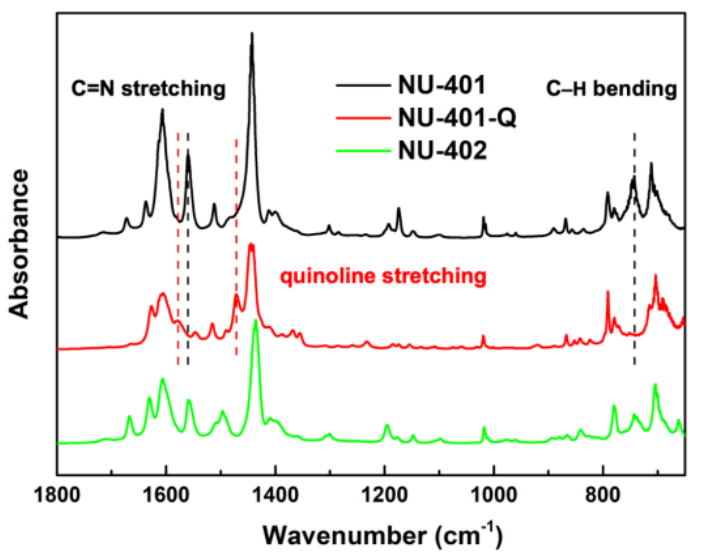

Figure 4. DRIFTS of NU-401, NU-401-Q and NU-402.

With the incorporation of phenylacetylene into the Schiff base bond, the stability of NU-401-Q compared with the original NU-401 was evaluated by immersing the materials in deionized water for 20 hours. NU401- $\mathrm{Q}$ with a more rigid $\mathrm{C}=\mathrm{N}$ bond fixated by phenylacetylene survived the aqueous environment while NU-401 completely lost its crystallinity and porosity (Figure 2 and Figure 5). After treating NU401-Q with deionized water, more than $75 \%$ of the BET area was retained (ca. 1610 vs. $2140 \mathrm{~m}^{2} / \mathrm{g}$ ) according to the nitrogen sorption isotherms. The hysteresis at the range of $\mathrm{P} / \mathrm{P}_{0}=0.5 \sim 0.8$ in the isotherms of water-treated NU-401-Q could be attributed to the defects inside the network where the uncoordinated $\mathrm{Zr}_{6}$ clusters and organic linkers were spontaneously dismissed under aqueous conditions (Figure 5). Apparently, the water-resistent ability of NU-401 was effectively improved after incorporation of the phenylacetylene. This proposed procedure could be scaled up to several grams in one batch with negligible influence on the quality of resulting materials.
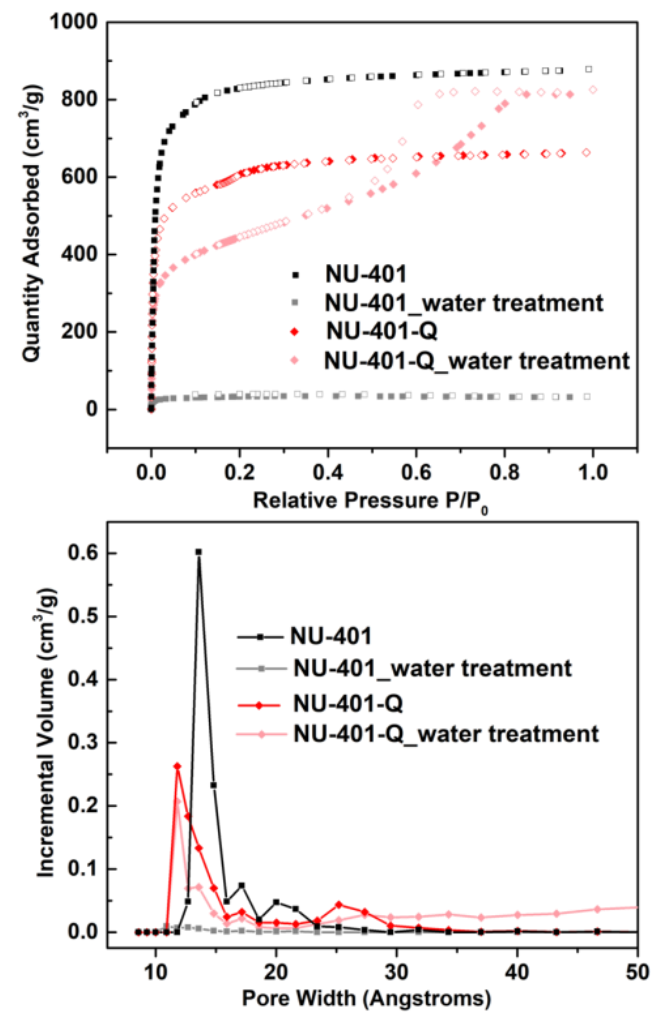

Figure 5. $\mathrm{N}_{2}$ sorption isotherms (up) and DFT-calculated pore size distribution (down) of NU-401, NU-401-Q and their corresponding materials after water treatment.

Oxygen storage is important for healthcare, industrial and military applications, and high porosity is one of the most critical factors for screening materials for oxygen storage. ${ }^{59}, 60$ For Zr-MOFs reported in this study which were prepared in one-pot and with high surface areas, the excess oxygen adsorption isotherms for NU-401, NU-402 and NU-401-Q were measured up to 100 bar at room temperature (Figure 6). NU-401 and NU-401-Q showed gravimetric oxygen uptakes of 8.4 and $8.8 \mathrm{~mol} / \mathrm{kg}$, respectively at $95 \mathrm{bar}$, comparable to previouly reported HKUST-1,59 while NU-402 showed a moderate oxygen capacity due to a lower 
pore volume. The isotherm data was fitted to the Toth model, 59 the parameters of which were summarized in the supporting information (Figure6, Table S2). Other than gravimetric capacity, volumetric oxygen uptake was more crucial, which is directly related to the size of the storage tank. By taking the void fraction and density into account, the volumetric uptake was calculated based on the ideal crystallographic density and plotted with increasing pressure. It was found that NU-401-Q had a higher volumetric capacity compared to the parent NU-401 (Figure 6, down), emphasizing the effectiveness of the post-synthetic strategy utilized here. For practical oxygen storage, deliverable oxygen adsorption capacity is critical to evaluate promising structures, which is defined as the uptake amount between storage pressure of 100 bar and release pressure of 5 bar. For the materials reported in this study, their deliverable capacities followed the similar trend as the excess uptakes reached up to $\sim 190 \mathrm{~cm}^{3} / \mathrm{cm}^{3}$ deliverable $\mathrm{O}_{2}$ uptake for NU-401-Q (Table S3).
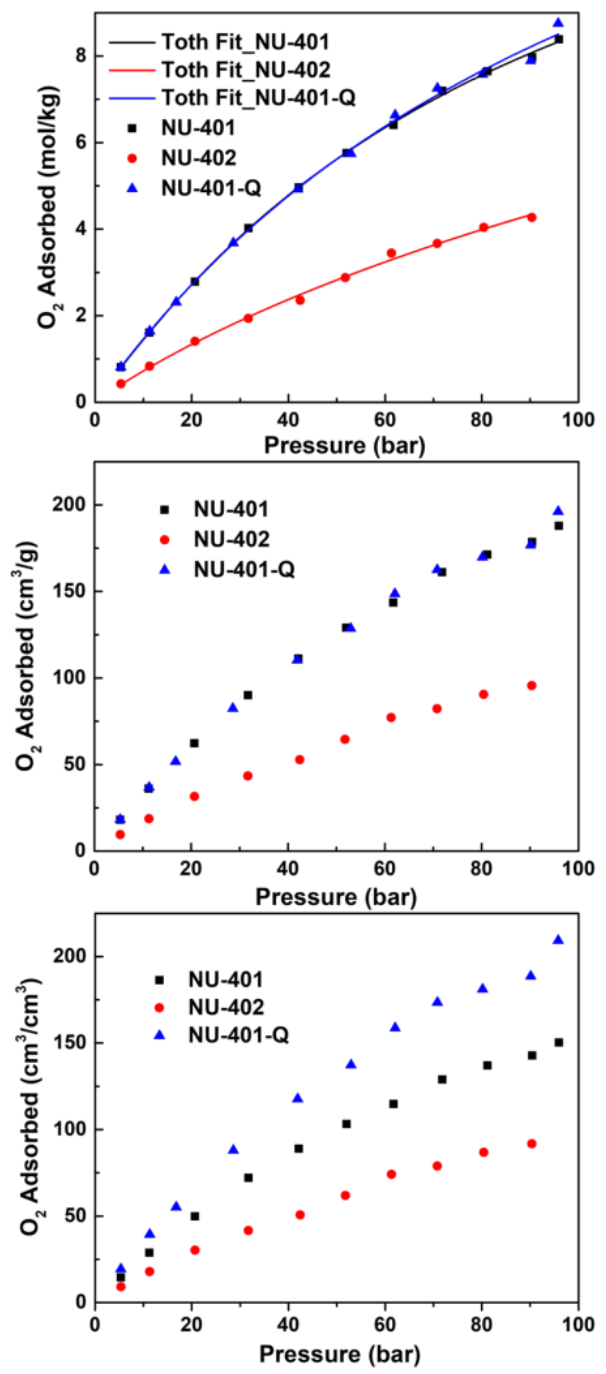

Figure 6. Toth model fitted (up), gravimetric (middle) and volumetric (down) excess $\mathrm{O}_{2}$ uptake on NU-401, NU-402 and NU-401-Q.
In conclusion, this study showed that access to high suface area Zr-MOFs built from ditopic ligands can be simplified to a methedology where linker synthesis and assembly of 3-D MOFs occurs in a one-pot fashion which is highly desirable for scalability. We demonstrated that highly porous Zr-MOFs with fcu topology can be prepared in a one-pot synthesis using benzoic acid with amine and aldehyde functionalities and Zr salt in DMF. Furthermore, with a simple AzaDiels-Alder addition, the hydrolytic stability of the resulting Zr-MOF was greatly enhanced. In addition, NU-401-Q showed an impressive volumetric $\mathrm{O}_{2}$ uptake at room temperature which exceed the parent material, NU-401.

\section{ASSOCIATED CONTENT}

Supporting Information

The Supporting Information is available free of charge on the ACS Publications website.

Crystallographic data for NU-401

Crystallographic data for NU-402

Crystallographic data for NU-401-Q (CIF)

Materials synthesis, Scanning Electron Microscope images, TGA curves, crystal structure modeling, Pawley refinement results, $\mathrm{O}_{2}$ adsorption experiment and analysis (PDF)

\section{AUTHOR INFORMATION}

Corresponding Author

*o-farha@northwestern.edu

Notes

The authors declare no competing financial interest.

\section{ACKNOWLEDGMENT}

O. K. F. gratefully acknowledges support from the Defense Threat Reduction Agency (HDTRA1-19-1-0007). This work made use of the EPIC facility of Northwestern University's NUANCE Center, which has received support from the Soft and Hybrid Nanotechnology Experimental (SHyNE) Resource (NSF NNCI-1542205); the MRSEC program (NSF DMR-1720139) at the Materials Research Center; the International Institute for Nanotechnology (IIN); the Keck Foundation; and the State of Illinois, through the IIN. This work made use of the IMSERC at Northwestern University, which has received support from the NSF (CHE-1048773 and DMR-0521267); Soft and Hybrid Nanotechnology Experimental (SHyNE) Resource (NSF NNCI1542205); the State of Illinois and International Institute for Nanotechnology (IIN). D.A.G.-G acknowledges funding from NSF CAREER (Award CBET 1846707).

\section{REFERENCES}

(1) Cooper, A. I.; Rosseinsky, M. J., Improving pore performance. Nat. Chem. 2009, 1, 26

(2) Shimizu, G. K. H.; Vaidhyanathan, R.; Taylor, J. M., Phosphonate and sulfonate metal organic frameworks. Chem. Soc. Rev. 2009, 38 (5), 1430-1449. 
(3) Yaghi, O. M.; O'Keeffe, M.; Ockwig, N. W.; Chae, H. K.; Eddaoudi, M.; Kim, J., Reticular synthesis and the design of new materials. Nature 2003, 423, 705-714.

(4) Takashima, Y.; Long, D.-L.; Cronin, L., Towards imaging electron density inside metal-organic framework structures. Chem. Commun. 2014, 50 (18), 2271-2274.

(5) Hönicke, I. M.; Senkovska, I.; Bon, V.; Baburin, I. A.; Bönisch, N.; Raschke, S.; Evans, J. D.; Kaskel, S., Balancing Mechanical Stability and Ultrahigh Porosity in Crystalline Framework Materials. Angew. Chem. Int. Ed. 2018, 57 (42), 13780-13783.

(6) Farha, O. K.; Özgür Yazaydın, A.; Eryazici, I.; Malliakas, C. D.; Hauser, B. G.; Kanatzidis, M. G.; Nguyen, S. T.; Snurr, R. Q.; Hupp, J. T., De novo synthesis of a metal-organic framework material featuring ultrahigh surface area and gas storage capacities. Nat. Chem. 2010, 2 944

(7) Nugent, P.; Belmabkhout, Y.; Burd, S. D.; Cairns, A. J.; Luebke, R.; Forrest, K.; Pham, T.; Ma, S.; Space, B.; Wojtas, L.; Eddaoudi, M.; Zaworotko, M. J., Porous materials with optimal adsorption thermodynamics and kinetics for CO2 separation. Nature 2013, 495, 80-84.

(8) Zheng, J.-J.; Kusaka, S.; Matsuda, R.; Kitagawa, S.; Sakaki, S. Characteristic Features of CO2 and CO Adsorptions to Paddle-Wheeltype Porous Coordination Polymer. J. Phys. Chem. C 2017, 121 (35) 19129-19139.

(9) Li, L.; Lin, R.-B.; Krishna, R.; Wang, X.; Li, B.; Wu, H.; Li, J.; Zhou W.; Chen, B., Flexible-Robust Metal-Organic Framework for Efficient Removal of Propyne from Propylene. J. Am. Chem. Soc. 2017, 139 (23), 7733-7736.

(10) Peng, Y.-L.; Pham, T.; Li, P.; Wang, T.; Chen, Y.; Chen, K.-J.; Forrest, K. A.; Space, B.; Cheng, P.; Zaworotko, M. J.; Zhang, Z., Robus Ultramicroporous Metal-Organic Frameworks with Benchmark Affinity for Acetylene. Angew. Chem. Int. Ed. 2018, 57 (34), 10971 10975.

(11) Zhang, S.-Y.; Jensen, S.; Tan, K.; Wojtas, L.; Roveto, M.; Cure, J.; Thonhauser, T.; Chabal, Y. J.; Zaworotko, M. J., Modulation of Water Vapor Sorption by a Fourth-Generation Metal-Organic Material with a Rigid Framework and Self-Switching Pores. J. Am. Chem. Soc. 2018, 140 (39), 12545-12552.

(12) Kalmutzki, M. J.; Diercks, C. S.; Yaghi, O. M., Metal-Organic Frameworks for Water Harvesting from Air. Adv. Mater. 2018, 30 (37) 1704304

(13) Belmabkhout, Y.; Bhatt, P. M.; Adil, K.; Pillai, R. S.; Cadiau, A.; Shkurenko, A.; Maurin, G.; Liu, G.; Koros, W. J.; Eddaoudi, M., Natural gas upgrading using a fluorinated MOF with tuned $\mathrm{H} 2 \mathrm{~S}$ and $\mathrm{CO} 2$ adsorption selectivity. Nat. Energy 2018, 3 (12), 1059-1066.

(14) Li, Y.; Yang, Z.; Wang, Y.; Bai, Z.; Zheng, T.; Dai, X.; Liu, S.; Gui, D.; Liu, W.; Chen, M.; Chen, L.; Diwu, J.; Zhu, L.; Zhou, R.; Chai, Z.; Albrecht-Schmitt, T. E.; Wang, S., A mesoporous cationic thoriumorganic framework that rapidly traps anionic persistent organic pollutants. Nat. Commun. 2017, 8 (1), 1354.

(15) Nugent, P. S.; Rhodus, V. L.; Pham, T.; Forrest, K.; Wojtas, L.; Space, B.; Zaworotko, M. J., A Robust Molecular Porous Material with High CO2 Uptake and Selectivity. J. Am. Chem. Soc. 2013, 135 (30), 10950-10953.

(16) Zhang, X.; Huang, Z.; Ferrandon, M.; Yang, D.; Robison, L.; Li, P.; Wang, T. C.; Delferro, M.; Farha, O. K., Catalytic chemoselective functionalization of methane in a metal-organic framework. Nat. Catal. 2018, 1 (5), 356-362.

(17) Li, Z.; Peters, A. W.; Bernales, V.; Ortuño, M. A.; Schweitzer, N. M.; DeStefano, M. R.; Gallington, L. C.; Platero-Prats, A. E.; Chapman, K. W.; Cramer, C. J.; Gagliardi, L.; Hupp, J. T.; Farha, O. K., Metal-Organic Framework Supported Cobalt Catalysts for the Oxidative Dehydrogenation of Propane at Low Temperature. ACS Cent. Sci. 2017, 3 (1), 31-38.

(18) Kim, C. R.; Uemura, T.; Kitagawa, S., Inorganic nanoparticles in porous coordination polymers. Chem. Soc. Rev. 2016, 45 (14), 3828 3845.

(19) Simon-Yarza, T.; Mielcarek, A.; Couvreur, P.; Serre, C., Drug Delivery: Nanoparticles of Metal-Organic Frameworks: On the Road to In Vivo Efficacy in Biomedicine. Adv. Mater. 2018, 30 (37), 1870281.

(20) Chen, Y.; Li, P.; Modica, J. A.; Drout, R. J.; Farha, O. K., AcidResistant Mesoporous Metal-Organic Framework toward Oral Insulin Delivery: Protein Encapsulation, Protection, and Release. J. Am. Chem. Soc. 2018, 140 (17), 5678-5681.
(21) Woellner, M.; Hausdorf, S.; Klein, N.; Mueller, P.; Smith, M. W. Kaskel, S., Adsorption and Detection of Hazardous Trace Gases by Metal-Organic Frameworks. Adv. Mater. 2018, 30 (37), 1704679.

(22) Zhang, X.; Saber, M. R.; Prosvirin, A. P.; Reibenspies, J. H.; Sun, L.; Ballesteros-Rivas, M.; Zhao, H.; Dunbar, K. R., Magnetic ordering in TCNQ-based metal-organic frameworks with host-guest interactions. Inorg. Chem. Front. 2015, 2 (10), 904-911.

(23) Chen, Z.; Hanna, S. L.; Redfern, L. R.; Alezi, D.; Islamoglu, T.; Farha, O. K., Reticular chemistry in the rational synthesis of functional zirconium cluster-based MOFs. Coord. Chem. Rev. 2019, 386, 32-49.

(24) Chen, Z.; Weseliński, Ł. J.; Adil, K.; Belmabkhout, Y.; Shkurenko, A.; Jiang, H.; Bhatt, P. M.; Guillerm, V.; Dauzon, E.; Xue, D.-X.; O'Keeffe, M.; Eddaoudi, M., Applying the Power of Reticular Chemistry to Finding the Missing alb-MOF Platform Based on the $(6,12)$-Coordinated EdgeTransitive Net. J. Am. Chem. Soc. 2017, 139 (8), 3265-3274.

(25) Islamoglu, T.; Goswami, S.; Li, Z.; Howarth, A. J.; Farha, O. K.; Hupp, J. T., Postsynthetic Tuning of Metal-Organic Frameworks for Targeted Applications. Acc. Chem. Res. 2017, 50 (4), 805-813.

(26) Tanabe, K. K.; Cohen, S. M., Postsynthetic modification of metalorganic frameworks - a progress report. Chem. Soc. Rev. 2011, 40 (2), 498-519.

(27) Li, T.; Kozlowski, M. T.; Doud, E. A.; Blakely, M. N.; Rosi, N. L., Stepwise Ligand Exchange for the Preparation of a Family of Mesoporous MOFs. J. Am. Chem. Soc. 2013, 135 (32), 11688-11691.

(28) Chen, Z.; Li, P.; Zhang, X.; Li, P.; Wasson, M. C.; Islamoglu, T.; Stoddart, J. F.; Farha, O. K., Reticular Access to Highly Porous acs-MOFs with Rigid Trigonal Prismatic Linkers for Water Sorption. J. Am. Chem. Soc. 2019, 141 (7), 2900-2905.

(29) Qin, J.; Yuan, S.; Zhang, L.; Li, B.; Du, D.-Y.; Huang, N.; Guan, W.; Drake, H.; Pang, J.; Lan, Y.-Q.; Alsalme, A.; Zhou, H.-C., Creating WellDefined Hexabenzocoronene in Zirconium Metal-Organic Framework by Postsynthetic Annulation. J. Am. Chem. Soc. 2019, 141 (5), 2054 2060.

(30) Vermeulen, N. A.; Karagiaridi, O.; Sarjeant, A. A.; Stern, C. L.; Hupp, J. T.; Farha, O. K.; Stoddart, J. F., Aromatizing Olefin Metathesis by Ligand Isolation inside a Metal- Organic Framework. J. Am. Chem Soc. 2013, 135 (40), 14916-14919.

(31) Chen, X.-M.; Tong, M.-L., Solvothermal in Situ Metal/Ligand Reactions: A New Bridge between Coordination Chemistry and Organic Synthetic Chemistry. Acc. Chem. Res. 2007, 40 (2), 162-170.

(32) Zhang, X.-M., Hydro(solvo)thermal in situ ligand syntheses. Coord. Chem. Rev. 2005, 249 (11), 1201-1219.

(33) Nguyen, H. L.; Gándara, F.; Furukawa, H.; Doan, T. L. H.; Cordova, K. E.; Yaghi, O. M., A Titanium-Organic Framework as an Exemplar of Combining the Chemistry of Metal- and Covalent-Organic Frameworks. J. Am. Chem. Soc. 2016, 138 (13), 4330-4333.

(34) J. Blake, A.; R. Champness, N.; S. M. Chung, S.; Li, W.-S.; Schröder, M., In situ ligand synthesis and construction of an unprecedented three-dimensional array with silver(i): a new approach to inorganic crystal engineering. Chem. Commun. 1997, (17), 1675-1676.

(35) Zheng, N.; Bu, X.; Feng, P., Self-Assembly of Novel Dye Molecules and $[\mathrm{Cd} 8(\mathrm{SPh}) 12] 4+$ Cubic Clusters into Three-Dimensional Photoluminescent Superlattice. J. Am. Chem. Soc. 2002, 124 (33), 96889689.

(36) Evans, 0. R.; Lin, W., Synthesis of Zinc Oxalate Coordination Polymers via Unprecedented Oxidative Coupling of Methanol to Oxalic Acid. Cryst. Growth Des. 2001, 1 (1), 9-11.

(37) Hu, S.; Chen, J.-C.; Tong, M.-L.; Wang, B.; Yan, Y.-X.; Batten, S. R., $\mathrm{Cu} 2+-$ Mediated Dehydrogenative Coupling and Hydroxylation of an $\mathrm{N}$ Heterocyclic Ligand: From Generation of a New Tetratopic Ligand to the Designed Assembly of Three-Dimensional Copper(I) Coordination Polymers. Angew. Chem. Int. Ed. 2005, 44 (34), 5471-5475.

(38) Grigolo, T. A.; de Campos, S. D.; Manarin, F.; Botteselle, G. V.; Brandão, P.; Amaral, A. A.; de Campos, E. A., Catalytic properties of a cobalt metal-organic framework with a zwitterionic ligand synthesized in situ. Dalton Trans. 2017, 46 (45), 15698-15703.

(39) Debatin, F.; Thomas, A.; Kelling, A.; Hedin, N.; Bacsik, Z.; Senkovska, I.; Kaskel, S.; Junginger, M.; Müller, H.; Schilde, U.; Jäger, C.; Friedrich, A.; Holdt, H.-J., In Situ Synthesis of an Imidazolate-4amide-5-imidate Ligand and Formation of a Microporous Zinc-Organic Framework with H2-and CO2-Storage Ability. Angew. Chem. Int. Ed. 2010, 49 (7), 1258-1262.

(40) Wang, X.-L.; Zhang, R.; Wang, X.; Lin, H.-Y.; Liu, G.-C., An Effective Strategy To Construct Novel Polyoxometalate-Based Hybrids by 
Deliberately Controlling Organic Ligand Transformation In Situ. Inorg. Chem. 2016, 55 (13), 6384-6393.

(41) Han, Y.; Zheng, H.; Liu, K.; Wang, H.; Huang, H.; Xie, L.-H.; Wang, L.; Li, J.-R., In-Situ Ligand Formation-Driven Preparation of a Heterometallic Metal-Organic Framework for Highly Selective Separation of Light Hydrocarbons and Efficient Mercury Adsorption. ACS Appl. Mater. Interfaces 2016, 8 (35), 23331-23337.

(42) Zhu, H.-B.; Gou, S.-H., In situ construction of metal-organic sulfur-containing heterocycle frameworks. Coord. Chem. Rev. 2011, 255 (1), 318-338.

(43) Zhang, X.; Wang, Z.-J.; Chen, S.-G.; Shi, Z.-Z.; Chen, J-X.; Zheng, H.-G., Cd-Based metal-organic frameworks from solvothermal reactions involving in situ aldimine condensation and the highly sensitive detection of Fe3+ ions. Dalton Trans. 2017, 46 (7), 23322338.

(44) Zhao, H.; Qu, Z.-R.; Ye, H.-Y.; Xiong, R.-G., In situ hydrothermal synthesis of tetrazole coordination polymers with interesting physical properties. Chem. Soc. Rev. 2008, 37 (1), 84-100.

(45) Xu, Y.; Liu, W.; Li, D.; Chen, H.; Lu, M., In situ synthesized 3D metal-organic frameworks (MOFs) constructed from transition metal cations and tetrazole derivatives: a family of insensitive energetic materials. Dalton Trans. 2017, 46 (33), 11046-11052.

(46) Haikal, R. R.; Elmansi, A. M.; Ali, P.; Hassan, Y. S.; Alkordi, M. H., One pot synthesis of $\mathrm{Zr}$-carboxylate porous hybrid materials: orthogonal C-C heterocoupling and carboxylate-Zr assembly. RSC Adv 2016, 6 (48), 42307-42312.

(47) Kong, X.-J.; He, T.; Zhang, Y.-Z.; Wu, X.-Q.; Wang, S.-N.; Xu, M.M.; Si, G.-R.; Li, J.-R., Constructing new metal-organic frameworks with complicated ligands from "One-Pot" in situ reactions. Chem. Sci. 2019, $10,3949-3955$

(48) Anderson, R.; Gómez-Gualdrón, D. A., Increasing topological diversity during computational "synthesis" of porous crystals: how and why. CrystEngComm 2019, 21 (10), 1653-1665.

(49) Colon, Y. J.; Gomez-Gualdron, D. A.; Snurr, R. Q., Topologically Guided, Automated Construction of Metal-Organic Frameworks and Their Evaluation for Energy-Related Applications. Cryst. Growth Des. 2017, 17 (11), 5801-5810.

(50) Rappe, A. K.; Casewit, C. J.; Colwell, K. S.; Goddard, W. A.; Skiff, W. M., UFF, a full periodic table force field for molecular mechanics and molecular dynamics simulations. J. Am. Chem. Soc. 1992, 114 (25), 10024-10035.

(51) Materials Studio. Accelrys Software Inc., San Diego, CA 92121, USA., Accelrys Software Inc.

(52) Lippke, J.; Brosent, B.; von Zons, T.; Virmani, E.; Lilienthal, S.; Preuße, T.; Hülsmann, M.; Schneider, A. M.; Wuttke, S.; Behrens, P.; Godt, A., Expanding the Group of Porous Interpenetrated Zr-Organic Frameworks (PIZOFs) with Linkers of Different Lengths. Inorg. Chem. 2017, $56(2), 748-761$

(53) Schaate, A.; Roy, P.; Preuße, T.; Lohmeier, S. J.; Godt, A.; Behrens, P., Porous Interpenetrated Zirconium-Organic Frameworks (PIZOFs): A Chemically Versatile Family of Metal-Organic Frameworks. Chem. Eur. J. 2011, 17 (34), 9320-9325.

(54) Belowich, M. E.; Stoddart, J. F., Dynamic imine chemistry. Chem. Soc. Rev. 2012, 41 (6), 2003-2024.

(55) Liu, H.; Chu, J.; Yin, Z.; Cai, X.; Zhuang, L.; Deng, H., Covalent Organic Frameworks Linked by Amine Bonding for Concerted Electrochemical Reduction of CO2. Chem 2018, 4 (7), 1696-1709.

(56) Waller, P. J.; Lyle, S. J.; Osborn Popp, T. M.; Diercks, C. S.; Reimer, J. A.; Yaghi, O. M., Chemical Conversion of Linkages in Covalent Organic Frameworks. J. Am. Chem. Soc. 2016, 138 (48), 15519-15522.

(57) Dibble, D. J.; Umerani, M. J.; Mazaheripour, A.; Park, Y. S.; Ziller, J. W.; Gorodetsky, A. A., An Aza-Diels-Alder Route to Polyquinolines. Macromolecules 2015, 48 (3), 557-561.

(58) Li, X.; Zhang, C.; Cai, S.; Lei, X.; Altoe, V.; Hong, F.; Urban, J. J.; Ciston, J.; Chan, E. M.; Liu, Y., Facile transformation of imine covalent organic frameworks into ultrastable crystalline porous aromatic frameworks. Nat. Commun. 2018, 9 (1), 2998.

(59) DeCoste, J. B.; Weston, M. H.; Fuller, P. E.; Tovar, T. M.; Peterson, G. W.; LeVan, M. D.; Farha, O. K., Metal-Organic Frameworks for Oxygen Storage. Angew. Chem. Int. Ed. 2014, 53 (51), 14092-14095.

(60) Moghadam, P. Z.; Islamoglu, T.; Goswami, S.; Exley, J.; Fantham, M.; Kaminski, C. F.; Snurr, R. Q.; Farha, O. K.; Fairen-Jimenez, D., Computer-aided discovery of a metal-organic framework with superior oxygen uptake. Nat. Commun. 2018, 9 (1), 1378. 
Table of Contents artwork

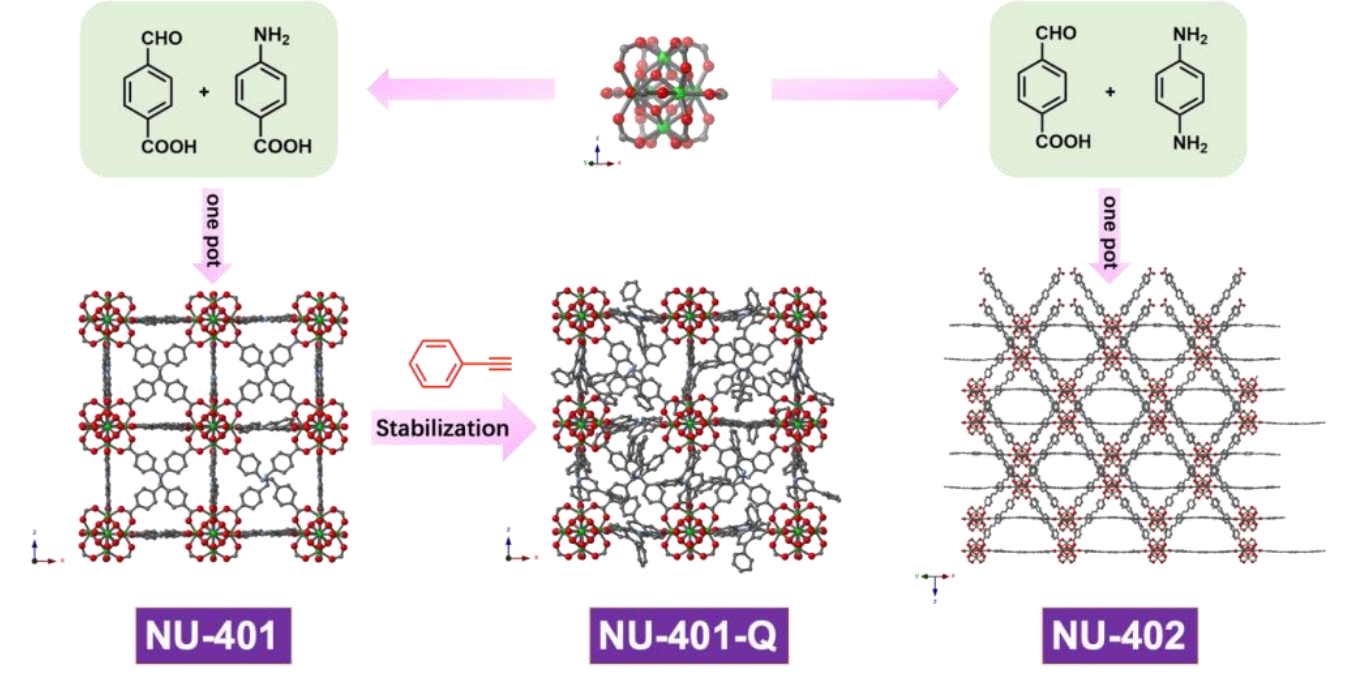

